\title{
Calculation Method of Stability Derivatives and Verification for Power-lift Aircraft
}

\author{
Shi Yazhou ${ }^{1}$ and Wang Weijun ${ }^{2, a}$ \\ ${ }^{1,2}$ School of Aeronautic Science and Engineering, Beihang University, Beijing, China
}

\begin{abstract}
It is difficult to realize the STOL and high-speed cruise at one time when designing an aircraft, whereas the application of power-lift technology has successfully solved this technical problem. The research about the dynamic characteristics of power-lift aircraft in hover and low speed flight regime is key important for the selection of control strategy in transition process. However, if adopting the traditional aerodynamic calculation method based on thrust coefficient concept, the hover and low speed forward flight of power-lift aircraft cannot be studied as a unified process and the stability derivatives cannot be accurately calculated in low speed. In this paper, a method for calculating the stability derivatives and model the aircraft by using full dimensional aerodynamic data is proposed, which can not only solve the problems mentioned above, and it is very convenient for the design of the control law in the transient process.
\end{abstract}

\section{Introduction}

The traditional calculation of the stability derivative is first calculating the dimensionless derivatives, and then translating the dimensionless derivatives into the dimensional derivatives by their relations [1]. This method is perfectly applicable to conventional aircraft, but it is not applicable for VTOL aircraft used power-lift technology under the conditions of hovering and low speed.

The traditional aerodynamic estimation method of power-lift aircraft is based on the concept of thrust coefficient $C_{T}\left(C_{T}=T\left(q^{*} S\right)^{-1}\right.$, $T$ is the engine thrust, dynamic pressure $q=\rho V^{2} / 2$, S is the reference area of the wing). The method can calculate aerodynamic forces accurately at the range of the specified thrust coefficient, however, due to there exists the problem of aerodynamic divergence under the condition of small velocity and large thrust coefficient [2], the dimensional stability derivatives calculated by the traditional method is inaccurate. Especially, when the aircraft is in hover, the traditional method can't be used. Therefore, when making the simulation of the power-lift aircraft with stability derivatives calculated by the traditional method, the dimensional stability derivatives of hovering, low-speed state and high-speed state will be discontinuous; this will influence the accuracy of the simulation.

In this paper, the dimensional aerodynamic data is directly used to solve the dimensional stability derivatives of the aircraft. The longitudinal linear time-varying simulation system for a STOL aircraft and a new type of VTOL aircraft is established, which can verify the effectiveness of the proposed method.

${ }^{\mathrm{a} C}$ Corresponding author : wangwj@buaa.edu.cn 


\section{Modeling method and the calculation of stability derivatives}

\subsection{Modeling method}

There are many aircraft dynamics modeling ways, such as nonlinear all-variables flight dynamic modeling, small disturbance linear modeling, linear time-varying simulation modeling [3-5]. In this paper, the dynamic model of the target aircraft is built by using a linear time-varying system. This method can convert the nonlinear dynamic equation into the linear time-varying dynamic equation, which can describe the motion process of aircraft accurately and simplify the design process of the aircraft control system when using the designing method of the linear control system.

The linear time-varying dynamics simulation of the aircraft starts from a baseline trim point, and a new benchmark is set at regular intervals. The time interval between any two-benchmark points is small enough to ensure that the changes of the state variables are within the range of small perturbations. The aerodynamic forces under arbitrary conditions can be calculated by using the following equations.

$$
\begin{aligned}
& X=X_{0}+\Delta X \\
& Z=Z_{0}+\Delta Z \\
& M=M_{0}+\Delta M
\end{aligned}
$$

$\mathrm{X}_{0}$ is the aerodynamic force at the benchmark, $\Delta \mathrm{X}$ is the aerodynamic force increment compared with the reference point. The calculation formula of aerodynamic increment is shown in equation (2).

$$
\begin{aligned}
& \Delta X=X_{\mathrm{u}} \Delta \mathrm{u}+X_{\mathrm{w}} \Delta \mathrm{w}+\mathrm{X}_{q} \Delta q+X_{\delta_{c}} \Delta \delta_{c}+X_{T} \Delta T \\
& \Delta Z=Z_{\mathrm{u}} \Delta \mathrm{u}+Z_{\mathrm{w}} \Delta \mathrm{w}+\mathrm{Z}_{q} \Delta q+Z_{\delta_{c}} \Delta \delta_{c}+Z_{T} \Delta T \\
& \Delta M=M_{\mathrm{u}} \Delta \mathrm{u}+M_{\mathrm{w}} \Delta \mathrm{w}+\mathrm{M}_{\mathrm{q}} \Delta \mathrm{q}+M_{\delta_{c}} \Delta \delta_{c}+M_{T} \Delta T
\end{aligned}
$$

Where $\delta_{\mathrm{c}}$ is the deflection angle of control surface.

Note that, $\mathrm{X}_{\mathrm{i}}, \mathrm{Z}_{\mathrm{i}}$ and $\mathrm{M}_{\mathrm{i}}(\mathrm{i}=u, w, q, \delta c, T)$ are the stability and control derivatives at the benchmark points, and their accuracy and continuity will affect the accuracy of the simulation results.

Finally, a longitudinal linear time-varying simulation model is shown in figure 1.

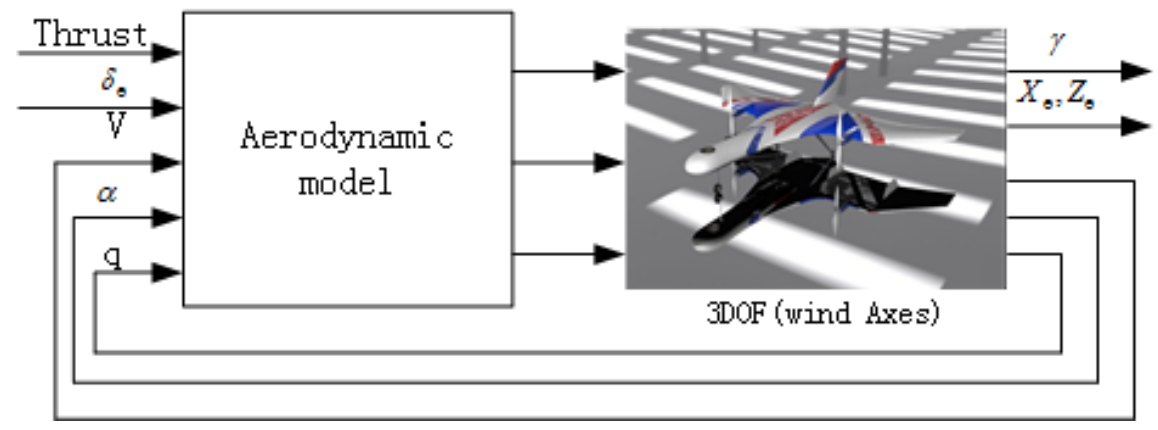

Figure 1. Linear time-varying simulation model of power-lift aircraft.

\subsection{Calculation of stability derivatives}

\subsubsection{Traditional calculation method}

Taking $\mathrm{X}_{\alpha}$ as an example, firstly the force coefficient $\mathrm{C}_{\mathrm{x}}$ about the $\mathrm{X}$-axis of the Body Axis system can be calculated by the following formula.

$$
C_{x}=C_{L} \alpha_{x}-C_{D}
$$


Where $C_{L}$ is the lift coefficient, $C_{D}$ is the drag coefficient.

Secondly, the dimensionless derivatives $\mathrm{C}_{\mathrm{X} \alpha}$ can be calculated by formula (4).

$$
C_{x \alpha}=\frac{\partial C_{x}}{\partial \alpha}=C_{L}+\alpha_{x} \frac{\partial C_{L}}{\partial \alpha}-\frac{\partial C_{D}}{\partial \alpha}
$$

When the stability axes are chosen as the Body Axis system, $\alpha_{x 0}=0$, the above formula can be transformed into:

$$
C_{x \alpha}=\left(\frac{\partial C_{x}}{\partial \alpha}\right)_{0}=C_{L 0}-\left(\frac{\partial C_{D}}{\partial \alpha}\right)_{0}
$$

Where the subscript zero indicates the reference flight condition.

Finally, $\mathrm{X}_{\alpha}$ can be obtained by formula (6).

$$
X_{\alpha}=C_{x \alpha} \times \frac{1}{2} \rho V^{2} S
$$

The stability derivatives $\mathrm{X}_{\alpha}$ calculated by the traditional method at high speed is quite accurate.

However, when the aircraft is in a low speed flight, especially, when the speed tends to zero, the corresponding thrust coefficient tends to infinity, which has exceeded its applicable range $\left(0.48 \leq \mathrm{C}_{\mathrm{T}} \leq 5.5\right)$.Therefore, a small velocity error will cause a huge deviation of the thrust coefficient $\mathrm{C}_{\mathrm{T}}$, which will cause the inaccurateness of $C_{x}[6-7]$. Therefore, the stability derivative calculated by the formula (5) and the formula (6) is also inaccurate.

In addition, when the aircraft is in hover, the inflow speed Va is 0 , the aerodynamic data of aircraft cannot be expressed in the form of dimensionless coefficients, so the traditional methods cannot be used.

\subsubsection{New calculation method.}

In order to unify the calculation method of the stability derivatives of aircraft under high-speed flight, low speed flight and hovering condition, the calculation method by using dimensional data is presented.

Taking $\mathrm{X}_{\alpha}$ as an example. The dimensional aerodynamic data under trim conditions of the aircraft is given in table 1 . Note that the engine thrust and its effects on aerodynamic forces have been included in the aerodynamic data.

Table 1. The axial force $\mathrm{X}$ of the aircraft vary as the angle of attack $\alpha$ and thrust $\mathrm{T}$.

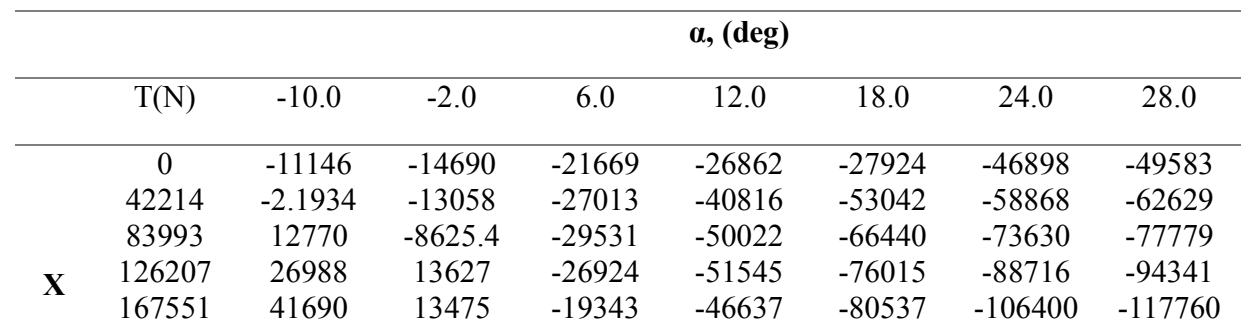

From the above table, supposing the trim thrust is 82191.38 , the relation between $\alpha$ and $\mathrm{X}$ is shown as figure 2 . 


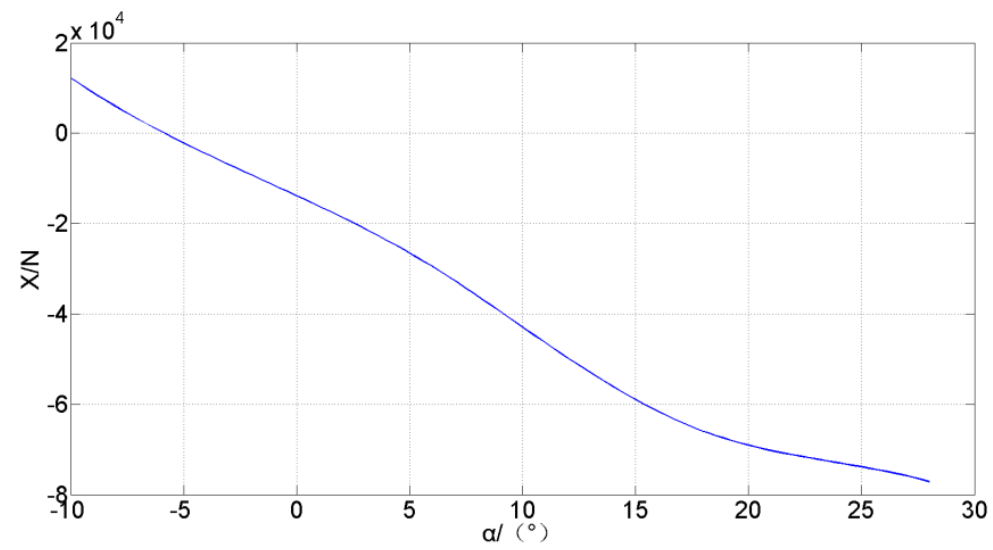

Figure 2. The curve about $X$ varying as $\alpha$.

Differentiating the curve in figure 2, figure 3 can be got as below.

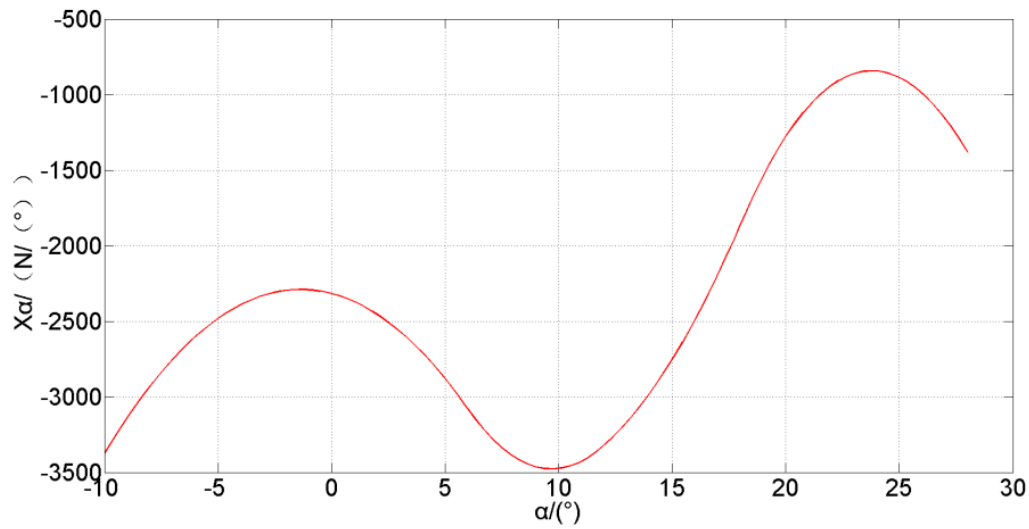

Figure 3. The curve about $X_{\alpha}$ varying as $\alpha$.

The value of the $X_{\alpha}$ of the aircraft at the trim angle of attack $\alpha$ can be obtained from figure 3 .

Other dimensional stability derivatives of aircraft, such as $\mathrm{u}$ derivatives, $\mathrm{q}$ derivatives, $\dot{\alpha}$ derivatives and so on, can be conveniently obtained by the above methods.

\section{Simulation verification}

\subsection{Simulation for a STOL aircraft}

In this part, a linear time-varying simulation of the EBF aircraft in reference [8] is made in Simulink, and a step response analysis compare with the step response curve(as shown in figure 4) in the reference is done.

The initial status parameters of the aircraft are selected the same as it in reference 8 , as shown in equation (7).

$$
\begin{aligned}
& V_{0}=36.01111 \mathrm{~m} / \mathrm{s}, \alpha_{0}=4.1550^{\circ}, \gamma_{0}=-6.5^{\circ}, T=82191.38 N, \delta_{\mathrm{ddc} 0}=5^{\circ} \\
& \delta_{\mathrm{sp} 0}=10^{\circ}, \delta_{\mathrm{h} 0}=-3.2084^{\circ}
\end{aligned}
$$

Where $\delta_{\mathrm{h}}$ is the deflection angle of horizontal tail, $\delta_{\mathrm{ddc}}$ is the deflection angle of direct drag control surface, $\delta_{\mathrm{sp}}$ is the deflection angle of spoiler. 

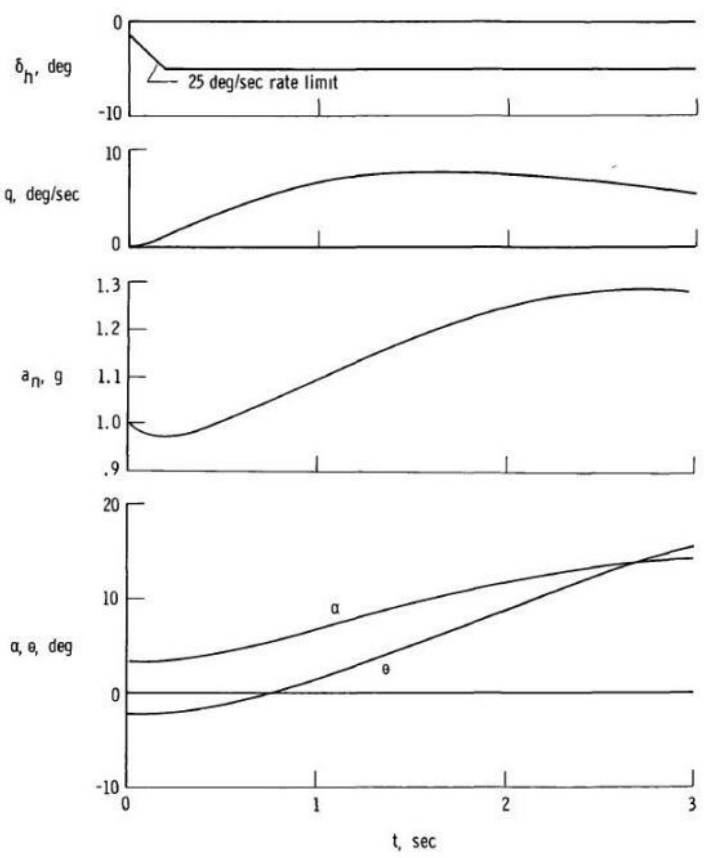

Figure 4. Time history of EBF airplane response to a longitudinal control column step input.

Deflecting the horizontal tail from reference point to $-7^{\circ}$, the step response curves generated from the simulation is shown in figure 5 .
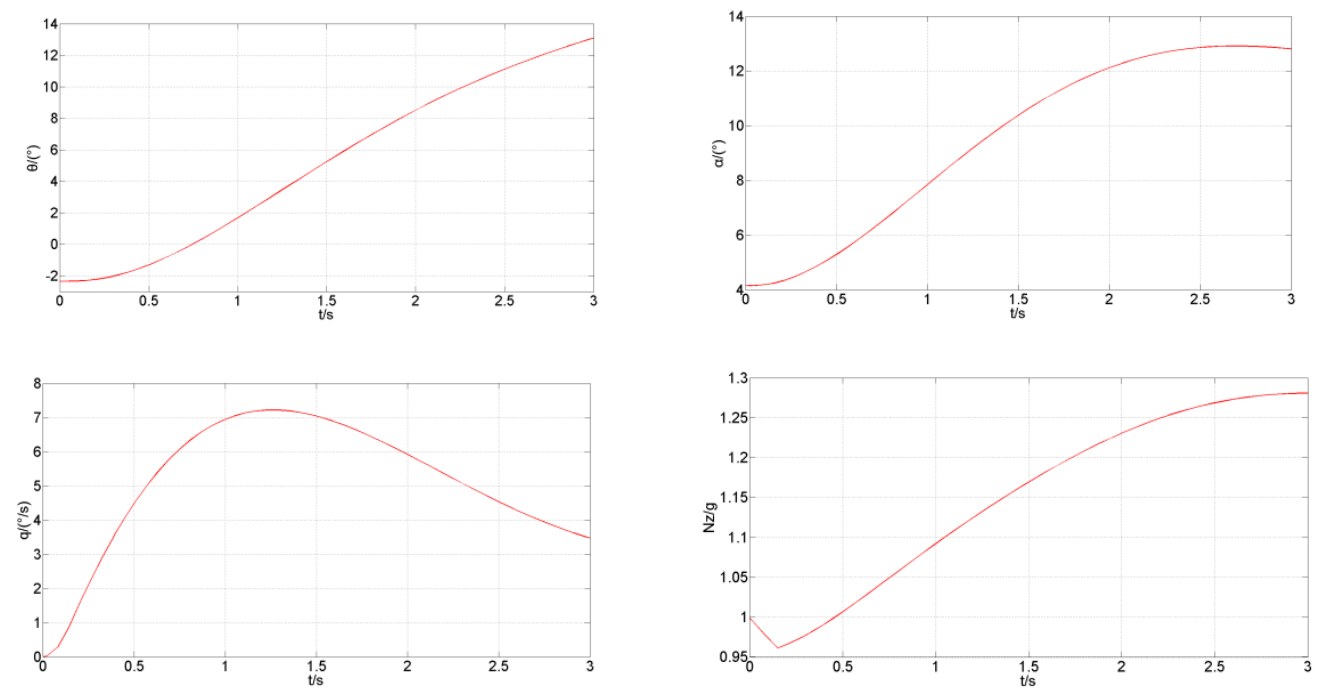

Figure 5. Step response curves of linear time-varying simulation.

Contrasting figure 4 and figure 5 , it is found that either the magnitudes or the trends of the two curves are basically consistent. Considering the influence of other factors, the result is ideal, which proves the correctness of the calculation method of stability derivatives proposed in this paper. 
Next, a simulation is made for a new type of VTOL power-lift aircraft. The configuration of the aircraft is shown in figure 6 .

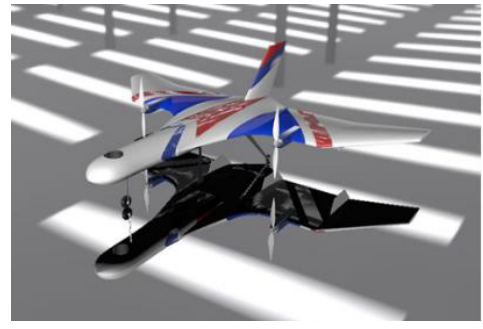

Figure 6. The configuration of the VTOL power-lift aircraft.

The aircraft can obtain the Lift-Enhancement by using the flap to deflect the propeller slipstream, but this will cause the serious coupling of aerodynamics and engine forces. Therefore, the acquisition and processing of aerodynamic data is essential. Nevertheless, the wind tunnel test with power system is very complex and costly, and the CFD calculation method [9-11] with power system is also complex. Here we use the below method to integrate the aerodynamic forces and engine forces.

Take the calculation of lift $\mathrm{L}$ as an example. The individual aerodynamic data can be obtained by CFD calculation, and the direct forces generated by propeller slipstream can be obtained by ground test [12].The integrated lift $\mathrm{L}$ can be represented as the following form.

$$
L=\eta * L_{a}+L_{T}
$$

Where $L_{a}$ is the aerodynamic force, $L_{T}$ is the direct forces generated by propeller slipstream; $\eta$ is the engine efficiency factor, which indicates the influence of propeller slipstream on aerodynamic force. $\eta \geq 1$ and increases with the increase of the throttle. The other aerodynamic data of the aircraft can also be obtained by this method.

The modeling simulation method introduced in the 2.1 section is used. The initial states of the aircraft as shown in formula (9).

$$
V=0 \mathrm{~m} / \mathrm{s}, \quad \theta=45.6242^{\circ}, \text { Throttle }=0.9406, \quad \delta_{\mathrm{f}}=59.4847, T_{f}=24.9 \mathrm{~N}
$$

Where $\delta_{\mathrm{f}}$ is the deflection angle of the flaps, $\mathrm{T}_{\mathrm{f}}$ is the pulling force of the lift fan.

The step response of the aircraft as the throttle increases to 1 is shown in figure 7.The response process of each parameter is similar to the actual flight-test. It is proved that the stability derivative calculation method and simulation modeling method proposed in this paper can achieve the simulation of VTOL aircraft from hovering to low speed flight.
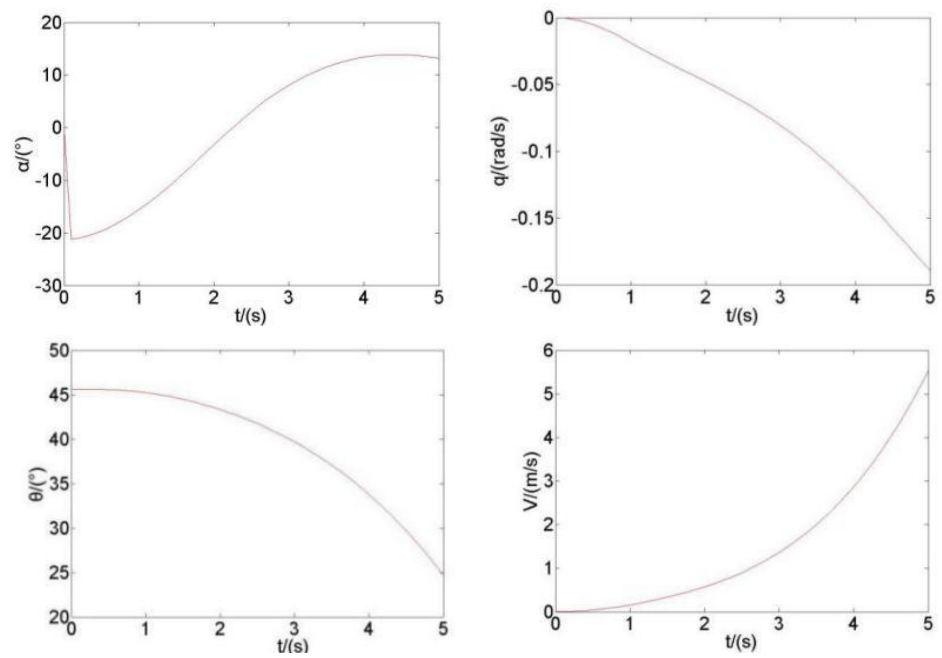

Figure 7. The step response of the VTOL power-lift aircraft. 


\section{Conclusion}

A method for calculating stability derivatives by using dimensional aerodynamic data is proposed and the linear time-varying simulation models are established to evaluate the accuracy of the derivatives calculation method and verify the effectiveness of the modeling method to unify the modeling process of VTOL aircraft from hovering to low speed flight.

The simulation results show that the method can avoid the inaccuracy of the stability derivatives due to the limitation of $\mathrm{C}_{\mathrm{T}}$ in the low speed flight. In addition, the method can unify the calculation method of stability derivatives at high speed, low speed and hovering condition, which maintains the continuity of stability derivatives, ensures the accuracy of the linear time-varying modeling and simulation method, and lays a solid foundation for the design of the subsequent longitudinal control system.

\section{Reference}

1. Etkin B and Teichmann T 1982 Dynamics of Flight: Stability and Control 3rd edition ed Cliff Robichaud and Catherine Beckham et al (New York: John Wiley \&Sons, Inc) chapter 5 pp $129-148$

2. Shengwei Z 2014 Research On Method of Powered Lift Aircraft Aerodynamics Calculation [J]. Aeronautical Science Technology, 2014(3):14-17.

3. Yue T, Wang L and Ai J 2013 Longitudinal Linear Parameter Varying Modeling and Simulation of Morphing Aircraft [J]. Journal of Aicraft, 2013, 50(6):1673-1681

4. Wang H, Dong X, Xue J, Liu J and Wang J 2016 Modeling and simulation of a time-varying inertia aircraft in aerial refueling [J].Chinese Journal of Aeronautics, 2016, 29(2):335-345

5. Johnson W, Samuel J, Craig IL and Ashkenas 1973 Analysis and moving base simulation of transition configuration management aspects of a powered lift aircraft NASA Technical Report by Ames Research Center, NASA-CR-114698, STI-TR-1015-2

6. Shengwei Z and Wei W 2017 Method for evaluating powered high-lift effects of externally blown flap [J]. Acta Aeronautica et Astronautica Sinica, 2017, 38(6): 220689-220689.

7. Bobbitt P and Margason R 2007 45th AIAA Aerospace Sciences Meeting and Exhibit, issue January(2007) pp 1-43

8. Bruce G. Powers and David A. Kier 1973 Simulator Evaluation of the low-speed flying qualities of an experimental STOL configuration with an externally blown flap wing or an augmentor wing NASA Flight Research Center Technical Note,NASA TN D-7454. pp 21-40

9. Abbas J and Narducci R 2010 Analysis of CFD modeling techniques over the mv-22 tiltrotor [C]//Phoenix: American Helicopter Society 66th Annual Forum, May 11-13

10. HY Xu, ZY Ye and AM Shi 2012 Numerical Study of Propeller Slipstream Based on Unstructured Dynamic Overset Grids [J]. Journal of Aircraft, 2012,49 (2) :384-389.

11. Sweeten BC 2010 CFD Analysis of UAVS using VORSTAB, FLUENT, and Advanced Aircraft Analysis Software, (University of Kansas)

12. Yuxing Z 2017 Lateral Decoupling Control Design of VSTOL Joined-Wing (Beihang University) pp 24-32 